\title{
CORIOAMNIONITIS EN BIOPSIA POR CONGELACIÓN DE PLACENTAS EN PACIENTES CON RIESGO DE INFECCIÓN Y SU RELACIÓN CON MORBIMORTALIDAD DEL RECIÉN NACIDO
}

Oscar Mendoza MD*, Diana Arias MD**, José Luis Rojas MD***, Jessica Gómez MD***, Katherine Hernández MD***, Daniel Castro MD****, Lina Romero MD****, Juan Pablo Contreras MD****, Pedro Montoya MD*****, Jennifer Cifuentes, José Luis Molano, María Paula Peña ******

\section{Resumen}

Se busca determinar si el diagnóstico de corioamnionitis en biopsia por congelación de placenta se relaciona con desenlace desfavorable en el recién nacido. Materiales y métodos: cohorte prospectiva de gestantes con alta sospecha de corioamnionitis a quienes se les practicó biopsia por congelación de cordón y membranas con estudio histopatológico usual, y se relacionó con la morbimortalidad del recién nacido. Resultados: en 23 placentas con sospecha de corioamnionitis se hizo estudio por congelación y parafina; cuatro fueron positivos en ambos y 19 negativos. Cinco desarrollaron sepsis temprana, todos con diagnóstico clínico de corioamnionitis en sus madres, de los cuales tres fueron positivos en ambos estudios microscópicos. Conclusiones: se evaluó la sepsis temprana y la mortalidad en hijos de madres con corioamnionitis diagnosticada mediante biopsias por congelación y parafina, evidenciando una adecuada relación. El diagnóstico por congelación fue preciso y temprano, posicionándose como una posible herramienta diagnóstica que permite un abordaje temprano tanto materno como del neonato con infección potencial, para así disminuir los desenlaces adversos con impacto en la morbimortalidad neonatal.

Palabras clave: corioamnionitis, biopsia por congelación, sepsis, neonato.

Abreviaturas: RN, recién nacido (s).

\section{HISTOLOGICAL CHORIOAMNIONITIS IN PLACENTAL BIOPSY BY FROZEN SECTION IN PATIENTS AT INCREASED RISK OF INFECTION AND ITS ASSOCIATION WITH NEONATAL MORBIDITY AND MORTALITY}

\section{Abstract}

Objective: to determine if histological diagnosis of chorioamnionitis in placental biopsy by frozen section is associated with adverse neonatal outcome. Materials and Methods: prospective cohort study performed in pregnant women with high risk of developing chorioamnionitis associated with neonatal morbidity and

Fecha recibido: octubre 25 de 2013 - Fecha aceptado: marzo 4 de 2014.

* Profesor Asistente, Secretario Académico, Facultad de Medicina. Fundación Universitaria de Ciencias de la Salud. Hospital de San José. Bogotá DC, Colombia.

** Instructor asistente, Fundación Universitaria de Ciencias de la Salud, Hospital de San José. Bogotá DC, Colombia.

*** Residente de Patología, Fundación Universitaria de Ciencias de la Salud. Bogotá DC, Colombia.

\footnotetext{
**** Residente de Pediatría, Fundación Universitaria de Ciencias de la Salud. Bogotá DC, Colombia.

****** Servicio Social Obligatorio, División de Investigaciones, Fundación Universitaria de Ciencias de la Salud. Bogotá DC, Colombia.

******* Estudiantes de Semilleros de Investigación en Patología, Facultad de Medicina, Fundación Universitaria de Ciencias de la Salud. Bogotá DC, Colombia.
} 
mortality. A placental biopsy by frozen section of umbilical cord and membranes was conducted and processed by the usual techniques. Results: placental frozen and paraphin-fixed sections were conducted in 23 patients with clinical suspicion of chorioamnionitis; four were positive in both studies and 19 were negative. Five infants born to women with diagnosed chorioamnionitis developed early-onset sepsis, of which three were positive in both histological studies. Conclusions: early-onset sepsis and mortality was evaluated in neonates of mothers with chorioamnionitis diagnosed by frozen and paraphin-fixed sections, evidencing an adequate relationship. Diagnosis by frozen biopsy section was accurate and timely, and has become a diagnostic tool which allows an early approach both in mothers and infants with potential infection, to reduce adverse outcomes impacting neonatal morbidity and mortality.

Key words: chorioamnionitis, biopsy by frozen section, sepsis, neonate.

\section{Introduc ción}

Se define corioamnionitis como la inflamación aguda de las membranas fetales y del líquido amniótico por bacterias o micoplasmas, por lo regular en el marco de ruptura prematura de membranas asociada con parto pretérmino e inversamente relacionado con la edad gestacional y el peso al nacer. ${ }^{1-8}$ Entre 1 y $4 \%$ de todos los partos en Estados Unidos se complican con corioamnionitis, pero esta incidencia es difícil de estimar por la gran variedad de criterios clínicos y paraclínicos que se utilizan para su diagnóstico., ${ }^{2,8}$ Se reporta en parto pretérmino hasta $30 \%$ en países desarrollados y cerca del $75 \%$ de la morbimortalidad neonatal se relaciona con prematuridad. Existe evidencia histológica de corioamnionitis en cerca de $30 \%$ de las placentas a la semana 29 y alrededor de $60 \%$ en aquellas entre las 23 y 24 de edad gestacional. El manejo de estas pacientes y sus RN, supone unos costos en salud estimados en US\$ 26.2 billones por año, solo en EEUU. ${ }^{3,6,7}$ Por esta razón, la población maternofetal es un grupo de interés epidemiológico en países en desarrollo y conociendo la importancia de la detección temprana de la infección, se busca un manejo precoz en los pacientes con alta sospecha de corioamnionitis, para así disminuir la incidencia de secuelas tanto maternas como neonatales. ${ }^{6,7}$

Durante los últimos años se han estudiado varias técnicas y pruebas de laboratorio (niveles de interleucina IL-6, IL-8, reacción en cadena de la polimerasa, procalcitonina y FNT- $\alpha$ entre otras) para el estudio temprano de la infección neonatal., ${ }^{910}$ Aunque hasta el momento no hay evidencia contundente que sugiera cuál utilizar, dentro de estos la PCR (proteína $\mathrm{C}$ reactiva) ha probado ser útil por su bajo costo y amplia disponibilidad, mostrando una sensibilidad del $84 \%$ y una especificidad de $96 \%$ (según los puntos de corte utilizados). ${ }^{11}$ No obstante, esta prueba es aplicable solo desde las primeras seis horas de vida del $\mathrm{RN}$, y si se suma el proceso de recolección y transporte de la muestra, así como la obtención y análisis de resultados, se puede producir una demora indeseable en el diagnóstico, suficiente para que se instaure un proceso inflamatorio que conlleve a sepsis neonatal y constituya un factor de riesgo adicional. ${ }^{6,12,13}$

Por otra parte, la biopsia por congelación ha demostrado ser una prueba histológica de gran utilidad para la emisión de diagnósticos preliminares durante el momento quirúrgico, pues aporta información histológica valiosa de diversas lesiones en un corto tiempo ${ }^{14,15}$, lo cual disminuye el tiempo que tardan los estudios patológicos, pues cuando se hace en forma usual requieren pasos del procesamiento de los tejidos como la fijación (la solidificación de las muestras que puede tomar hasta dos semanas), el proceso de deshidratación, hidratación y parafinización, que suma al menos un día más al procesamiento del espécimen, el corte y la coloración.

Con el estudio por congelación de los tejidos se logran cortes en solo minutos sacrificando en forma parcial la resolución de las imágenes evaluadas al microscopio, lo cual puede ser mínimo si se compara con el gran beneficio temporal que se adquiere al tener un diagnóstico que cambie las conductas de los médicos tratantes de manera inmediata., Aunque no hay gran 
evidencia, esta podría ser aprovechada para determinar la existencia de procesos inflamatorios en la placenta, cordón y membranas fetales, y de esta forma reducir el tiempo diagnóstico en el paciente con potencial infección, logrando implementar un manejo temprano, con lo que se espera un impacto positivo al reducir los costos, la morbimortalidad, la estancia hospitalaria y el manejo de los pacientes que realmente requieren intervención, sin ser sometidos a riesgos adicionales.

Por esta razón, se pretende determinar el grado de acuerdo entre la biopsia por congelación y el estudio histológico de rutina en el diagnóstico de corioamnionitis y su relación con el desenlace en los RN (sepsis temprana y mortalidad).

\section{Materiales y métodos}

Se describe una serie de casos de pacientes del Hospital de San José de Bogotá DC, en la cual se incluyeron madres con sospecha de corioamnionitis, basados en la presencia de al menos dos de los criterios de Gibbs ${ }^{16}$ : temperatura materna $>37,8^{\circ} \mathrm{C}$, taquicardia materna $>100$ latidos por minuto, taquicardia fetal > 160, olor fétido del líquido amniótico, hipersensibilidad uterina y leucocitosis materna (recuento $>15.000$ leucocitos por microlitro.

Asimismo, se incluyeron los casos con antecedente materno de ruptura prematura de membranas (RPM) mayor de 18 horas sin importar la edad gestacional, los RN pretérmino (parto antes de la semana 34 por cualquier causa) y los RN con potencial infección previa recolección del consentimiento informado para la participación en el estudio, entre el primero de julio de 2011 y el 30 de junio de 2012.

Se excluyeron los casos de placentas sin cadena de frío por más de doce horas, las tuvieran tratamiento antibiótico materno por más de 24 horas previo al parto, presentaran muerte materna o fetal durante el seguimiento y aquellos con muerte neonatal antes del diagnóstico.

Posterior al alumbramiento, las placentas se enviaron al servicio de patología para el estudio histopatológico por congelación. Se realizó la descripción macroscópica: peso de la placenta, medición del plato corial, membranas y cordón umbilical, obtención y lectura de muestras para congelación (cortes de membranas ovulares y cortes de cordón umbilical), por último se procesó el resto de la placenta según el protocolo habitual (fijación en formol y corte en bloques de parafina). Las placentas fueron estudiadas por el patólogo correspondiente sin ser revisadas por quien realizó la biopsia por congelación (Figura 1)

Se consignó toda la información de ambos tipos de procesamiento (estudios histopatológicos por congelación y parafina) en el instrumento de recolección de datos del servicio de patología, sumado al diagnóstico histológico o no de corioamnionitis. A los RN incluidos, según el protocolo del servicio de neonatología, se les tomaron cuadro hemático y PCR (proteína $\mathrm{C}$ reactiva) a las seis horas de vida y hemocultivos (en caso de diagnóstico positivo) para inicio del manejo antibiótico. En este estudio se continuó con el seguimiento de siete días a los RN con PCR negativa para descartar morbilidades secundarias y así dar de alta a aquellos con bajo riesgo de sepsis neonatal. Se consignaron los resultados en el formato del servicio de pediatría, donde en cada caso se evaluó sin conocer la identidad de los pacientes evitando conocer el resultado del estudio de patología, cumpliendo con los protocolos de detección y tratamiento avalados por el servicio.

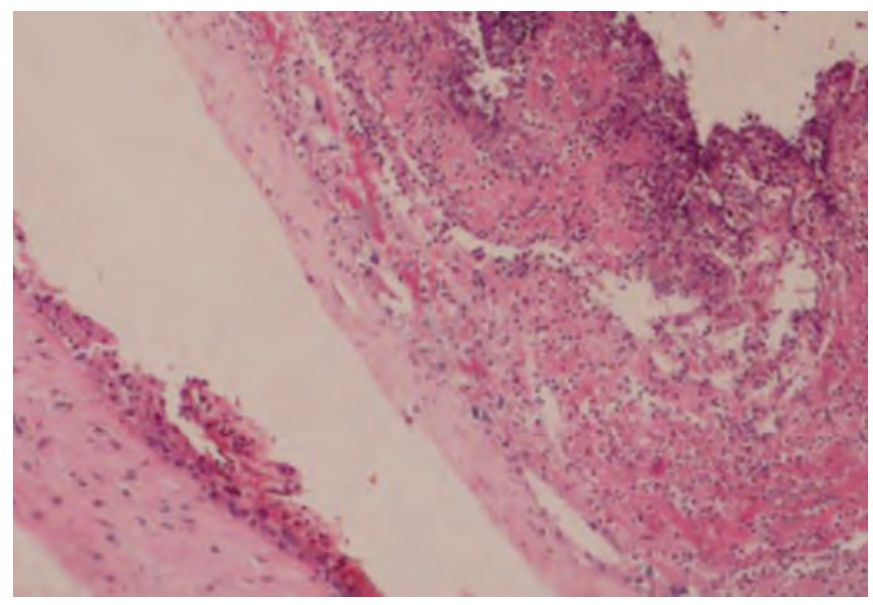

Figura I. Infiltrado inflamatorio de tipo polimorfonuclear neutrófilo en las membranas amnióticas con lo que se realiza el diagnóstico histológico de corioamnionitis. 
Se construyó una base de datos en Microsoft excel $2010 @$ y se analizaron en Stata12@. Las variables con medidas de tendencia central y de dispersión (variables cuantitativas), y con frecuencias relativas y absolutas (variables cualitativas). El estudio fue aprobado por el comité de ética en investigación con seres humanos del Hospital de San José de Bogotá DC, Colombia.

\section{Resultados}

Cumplieron los criterios de inclusión 23 pacientes con sospecha de corioamnionitis. La edad gestacional (EG) promedio fue de 33.6 semanas (DE: 3.6). El 56.5\% fueron neonatos con EG $\leq 34$ semanas. En el $65.2 \%$ no se halló evidencia de RPM (Tabla 1). Todos los casos fueron llevados a estudio patológico de parafina y a biopsia por congelación. Al comparar los dos estudios se encontraron cuatro casos positivos en ambos y 19 negativos en ambos, otorgándole un porcentaje de acuerdo del $100 \%$ a la biopsia por congelación con el estudio en parafina (Tabla 2).

Frente a los otros métodos diagnósticos como los criterios de Gibbs para detección de corioamnionitis materna, se presentaron seis casos positivos, tres de los cuales fueron confirmados por estudio patológico de rutina y biopsia por congelación y uno en el cual la clínica fue negativa pero con resultado positivo en las biopsias por congelación y parafina.

\begin{tabular}{|l|c|}
\hline \multicolumn{2}{|c|}{ Tabla I. Características de la población } \\
\hline Características & n (\%) \\
\hline Total & 23 \\
\hline Edad gestacional, promedio (DE) & $33,6^{*}(3.6)$ \\
\hline menor de 34 semanas & $13(56.5)$ \\
\hline mayor de 34 semanas & $10(43.5)$ \\
\hline RPM & $8(34.8)$ \\
\hline RPM I2-I8 horas & $4(17.3)$ \\
\hline RPM I9-24 horas & $3(13)$ \\
\hline RPM mayor de 24 & $1(4.3)$ \\
\hline Sin evidencia de RPM & $15(65.2)$ \\
\hline Corioamnionitis materna & $6(26)$ \\
\hline Taquicardia fetal & $1(4.3)$ \\
\hline
\end{tabular}

RPM: Ruptura prematura de membranas.
Se encontraron $19 \mathrm{RN}$ con alguna patología como sepsis neonatal, neumonía y muerte. De los nueve con sepsis neonatal, temprana, todos tuvieron diagnóstico clínico materno de corioamnionitis y en tres de ellos el resultado fue positivo en las biopsias por congelación y parafina. Dos pacientes fueron negativos en ambos estudios (Tabla 3).

\section{Disc usión}

El presente estudio evidencia un alto nivel de acuerdo entre congelación e histología de rutina para el diagnóstico de corioamnionitis ${ }^{14,15}$, posicionando así a la primera biopsia como una posible herramienta diagnóstica para la detección precoz de la infección materna, lo cual permite realizar un abordaje temprano del RN potencialmente infectado para disminuir los desenlaces adversos, generando un impacto en la morbimortalidad neonatal y materna.

Se observó además que el resultado de la biopsia por congelación y la parafina se relacionan con la ocurrencia de sepsis temprana en el RN, pues hay alta correspondencia entre el número de los que tuvieron resultados positivos en la congelación y la parafina. La correspondencia también fue clave en la ausencia de sepsis y resultados negativos, lo

\begin{tabular}{|c|c|c|c|}
\hline \multirow{2}{*}{$\begin{array}{l}\text { Biopsia por } \\
\text { congelación }\end{array}$} & \multicolumn{2}{|c|}{ Histología } & \multirow{2}{*}{ Total } \\
\hline & Si & No & \\
\hline Si & 4 & 0 & 4 \\
\hline No & 0 & 19 & 19 \\
\hline Total & 4 & 19 & 23 \\
\hline
\end{tabular}

\begin{tabular}{|c|c|c|c|c|}
\hline \multicolumn{5}{|c|}{$\begin{array}{r}\text { Tabla 3. Corioamnionitis } \\
\text { temprana }\end{array}$} \\
\hline \multirow{2}{*}{\multicolumn{2}{|c|}{$\begin{array}{l}\text { Diagnóstico de } \\
\text { corioamnionitis }\end{array}$}} & \multicolumn{2}{|c|}{$\begin{array}{l}\text { Sepsis neonatal temprana } \\
(\mathrm{n})\end{array}$} & \multirow{2}{*}{\begin{tabular}{c|} 
Total \\
23
\end{tabular}} \\
\hline & & $\mathrm{Si}(\mathrm{n}=5)$ & No $(n=\mid 8)$ & \\
\hline \multirow{2}{*}{ Por clínica } & $\mathrm{Si}$ & 5 & I & 6 \\
\hline & No & 0 & 17 & 17 \\
\hline \multirow{2}{*}{$\begin{array}{l}\text { Por histología/ } \\
\text { parafina }\end{array}$} & Si & 3 & I & 4 \\
\hline & No & 2 & 17 & 19 \\
\hline \multirow{2}{*}{$\begin{array}{l}\text { Por biopsia por } \\
\text { congelación }\end{array}$} & $\mathrm{Si}$ & 3 & 1 & 4 \\
\hline & No & 2 & 17 & 19 \\
\hline
\end{tabular}


cual podría apoyar la hipótesis de que la biopsia por congelación sirve como herramienta diagnóstica para comenzar el manejo temprano, pues en la actualidad solo se inicia tras tener resultados de las pruebas estándares (PCR, hemocultivos), impactando de forma directa en la disminución de desenlaces adversos tempranos.

Se resalta que debido a que en este estudio se realizó un seguimiento del RN hasta los siete días de vida, pudieron ocurrir desenlaces adversos sin relación directa con la corioamnionitis materna, debido a que los riesgos inherentes a la hospitalización e invasión del RN por el soporte ventilatorio y hemodinámico requeridos aumentan la aparición de infecciones adquiridas después del parto; por ello se decidió analizar solo los que presentaron sepsis neonatal temprana.

En cuanto a la relación existente entre los estudios histológicos y las pruebas de rutina realizadas a los neonatos (hemocultivo PCR, y la relación de leucocitos maduros e inmaduros) se nota que los análisis aun requieren mayor valor estadístico dado el número limitado de casos del presente estudio (Tabla 4), por lo cual sería ideal uno multicéntrico con los dos hospitales con los que cuenta nuestra institución.

Hay que resaltar que con los hallazgos descritos en la presente investigación sumado a lo poco invasivo de la biopsia por congelación, así como a su rápido resultado y bajo costo, se puede recomendar para el diagnóstico de corioamnionitis, que sumado a las pruebas de rutina permite instaurar el manejo temprano en dicha población.

\section{Referencias}

1. de Sa D. Organisms implicated in chorioamnionitis?. Hum Pathol. 2006;37 (9): 1240.

2. Tita AT, Andrews WW. Diagnosis and management of clinical chorioamnionitis Clin Perinatol. 2010. p. 339-54.

3. Strunk T, Doherty D, Jacques A, Simmer K, Richmond P, Kohan R, et al. Histologic chorioamnionitis is associated with reduced risk of late-onset sepsis in preterm infants. Pediatrics. 2012 Jan; 129(1):e134-41.

4. Espitia-De La Hoz FJ. Diagnóstico y tratamiento de la corioamnionitis clínica. Rev Colomb Obstet Ginecol. 2008; 59(3):231-7.

\begin{tabular}{|c|c|c|c|}
\hline \multirow{2}{*}{$\begin{array}{l}\text { Método } \\
\text { diagnóstico }\end{array}$} & \multicolumn{2}{|c|}{$\begin{array}{l}\text { Evidencia de } \\
\text { corioamnionitis* }\end{array}$} & \multirow[t]{2}{*}{ Total } \\
\hline & SI & No & \\
\hline Hemocultivos & $\mathrm{n}$ & $\mathrm{n}$ & 23 \\
\hline positivo & 0 & 1 & 1 \\
\hline negativo & 4 & 18 & 22 \\
\hline \multicolumn{4}{|l|}{ PCR** } \\
\hline negativo & 2 & 13 & 15 \\
\hline positivo & 2 & 6 & 8 \\
\hline \multicolumn{4}{|l|}{ Índice de Oski**** } \\
\hline positivo & 2 & 1 & 3 \\
\hline negativo & 2 & 18 & 20 \\
\hline Leucocitos (> 35000) & 0 & 1 & I \\
\hline
\end{tabular}

*Diagnóstico histológico **PCR: proteína C reactiva; ***índice de Oski: recuento de células inmaduras / recuento de leucocitos totales.

5. Bersani I, Thomas W, Speer CP. Chorioamnionitis--the good or the evil for neonatal outcome?. J Matern Fetal Neonatal Med. 2012 Apr;25 Suppl 1:12-6.

6. Torricelli M, Voltolini C, Conti N, Vellucci FL, Orlandini C, Bocchi C, et al Histologic chorioamnionitis at term: implications for the progress of labor and neonatal wellbeing. J Matern Fetal Neonatal Med. 2013 Jan;26(2):188-92.

7. Cobo T, Kacerovsky M, Palacio M, Hornychova H, Hougaard DM, Skogstrand $\mathrm{K}$, et al. A prediction model of histological chorioamnionitis and funisitis in preterm prelabor rupture of membranes: analyses of multiple proteins in the amniotic fluid. J Matern Fetal Neonatal Med. 2012 Oct; 25(10):1995-2001.

8. Martinelli P, Sarno L, Maruotti GM, Paludetto R. Chorioamnionitis and prematurity: a critical review. J Matern Fetal Neonatal Med. 2012 Oct;25 Suppl 4:29-31.

9. Malik A, Hui CP, Pennie RA, Kirpalani H. Beyond the complete blood cell count and $\mathrm{C}$-reactive protein: a systematic review of modern diagnostic tests for neonatal sepsis. Arch Pediatr Adolesc Med. 2003;157(6):511-6.

10. Nq PC, Lam HS. Biomarkers for late-onset neonatal sepsis: cytokines and beyond. Clin Perinatol. 2010;37(3):599-610.

11. Nq PC, Cheng SH, Chui KM, Fok TF, Wong MY, Wong W, et al. Diagnosis of late onset neonatal sepsis with cytokines, adhesion molecule, and C-reactive protein in preterm very low birthweight infants. Arch Dis Child Fetal Neonatal Ed. 1997;77(3):F221-7.

12. Trochez-Martinez RD, Smith P, Lamont RF. Use of C-reactive protein as a predictor of chorioamnionitis in preterm prelabour rupture of membranes: a systematic review. BJOG. 2007;114(7):796-801.

13. van de Laar R, van der Ham DP, Oei SG, Willekes C, Weiner CP, Mol BW Accuracy of C-reactive protein determination in predicting chorioamnionitis and neonatal infection in pregnant women with premature rupture of membranes: a systematic review. Eur J Obstet Gynecol Reprod Biol. 2009;147(2):124-9.

14. Schmidt RL, Hunt JP, Hall BJ, Wilson AR, Layfield LJ. A systematic review and meta-analysis of the diagnostic accuracy of frozen section for parotid gland lesions. Am J Clin Pathol. 2011Nov; 136(5):729-38.

15. Liu LC, Lang JE, Lu Y, Roe D, Hwang SE, Ewing CA, et al. Intraoperative frozen section analysis of sentinel lymph nodes in breast cancer patients: a metaanalysis and single-institution experience. Cancer. 2011; 117(2):250-8.

16. Gibbs RS, Dinsmoor MJ, Newton ER, Ramamurthy RS. A randomized trial of intrapartum versus immediate postpartum treatment of women with intra-amniotic infection. Obstet Gynecol. 1988; 72(6):823-8. 